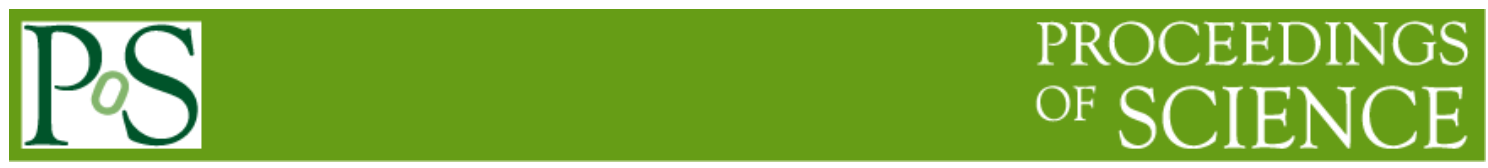

\title{
Measurement of the mass of the $\mathrm{W}$ boson at $\mathrm{D} \varnothing$
}

\author{
Tibor Kurča ${ }^{1}$ \\ IPNL, Université de Lyon, Université Lyon 1, CNRS/IN2P3 \\ 4 Rue E. Fermi 69622 Villeurbanne, France \\ E-mail: kurcalin2p3.fr
}

We present a measurement of the mass of the $\mathrm{W}$ boson using $\mathrm{D} \emptyset$ detector and half of the full Run II Fermilab Tevatron collider data corresponding to $5.3 \mathrm{fb}^{-1}$ of integrated luminosity collected at $\sqrt{\mathrm{s}}_{\mathrm{s}}=1.96 \mathrm{TeV}$. This result $M_{W}=80.375 \pm 0.023 \mathrm{GeV}$ is the combination of measurements with two independent data sets, the earlier DØ result with $1 \mathrm{fb}^{-1}$ [1] and the current analysis with $4.3 \mathrm{fb}^{-1}$ [2]. Decays of the $W \rightarrow e v$ are selected and the transverse momentum of the electron $p_{T}^{e}$, the transverse momentum of neutrino $p_{T}^{v}$, the transverse mass of the $\mathrm{W}$ boson $m_{T}^{W}$, and other new variables are used in the extraction of the $\mathrm{W}$ mass. With samples of 1677394 and 54512 candidate events in $W \rightarrow e v$ resp. $Z \rightarrow e e$ (both electrons at $|\eta|<1.05)$ channels we measured the $\mathrm{W}$ mass $M_{W}=80.367 \pm 0.026 \mathrm{GeV}$.

The European Physical Society Conference on High Energy Physics

18-24 July, 2013

Stockholm, Sweden

\footnotetext{
${ }^{1}$ Speaker for DØ collaboration
} 


\section{Introduction}

Precise measurement of the $\mathrm{W}$ boson mass $M_{W}$ itself as well as its relationship [1] with the masses of the top quark $M_{t}$ and that of Higgs boson $M_{H}$ are important tests of the standard model (SM). At higher orders of the SM electroweak (EW) theory the observables contain quantum loop corrections from virtual effects. Significant radiative corrections $\Delta \mathrm{r}$ of $M_{W}$ are comming from the top quark $\left(\Delta \mathrm{r} \sim M_{t}^{2}\right)$ and Higgs loops $\left(\Delta \mathrm{r} \sim \ln M_{H}\right)$ (Fig.1). The precise $M_{W}$ and $M_{t}$ measurements constrain the mass of Higgs $M_{H}$ (and vice-versa). Taking into account the current Tevatron average of $\Delta M_{t}=0.87 \mathrm{GeV}$ [3], for equivalent contribution to $M_{H}$ constraints the needed $\Delta M_{W}$ measurement precision is about $5 \mathrm{MeV}\left(\Delta M_{W} \sim 0.006^{*} \Delta M_{t}\right)$. The current world average is $\Delta M_{W}=15 \mathrm{MeV}$. It means that limiting factor here is $\Delta M_{W}$ precision and not that of top quark $\Delta M_{t}$.

Additional loops can be generated in SM extensions, e.g. in SUSY (Fig.1), and constraints inconsistent with direct searches would indicate new physics.

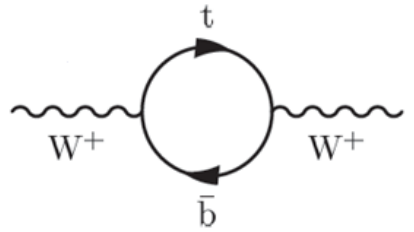

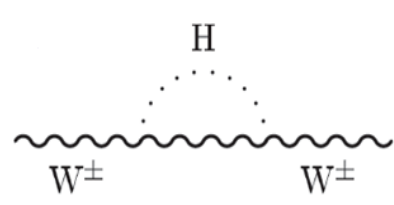

Fig.1

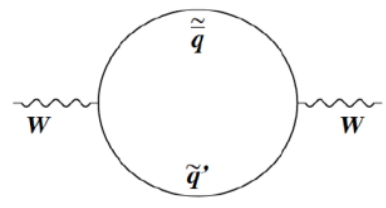

\subsection{Event Signatures}

In the $p \bar{p}$ interactions at Tevatron the quark-antiquark annihilation dominates and gluons may be radiated from quarks in initial state. In addition photons may be radiated from quarks and leptons in initial resp. in final states. The signature of $W \rightarrow e v$ events (Fig.2) is isolated electron with high $p_{T}^{e}$, the missing transverse momentum $\mathbb{E}_{T}$ due to the neutrino and the hadronic recoil energy. The calibration $Z \rightarrow e e$ sample has instead of a missing energy, the second high $p_{T}^{e}$ electron. Clean $\mathrm{W}$ samples with relatively low backgrounds $(1.02 \%$ multijet events, $1.08 \% Z \rightarrow e e$ and $1.67 \% W \rightarrow \tau v \rightarrow e v v v$ events) are selected from the data. But the requirements on the energy measurement precision are challenging: $0.02 \%$ and $\sim 1 \%$ for the electron energy resp. the hadronic recoil energy.

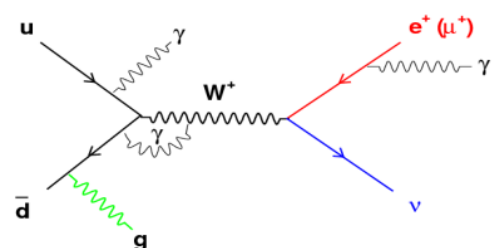

Fig.2

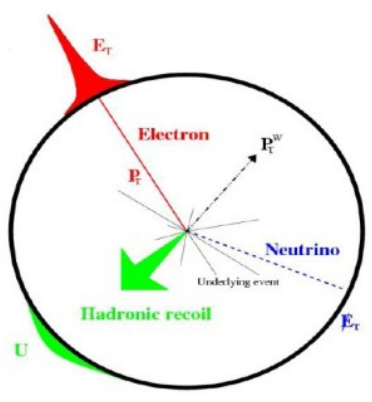




\subsection{Experimental Observables}

Longitudinal components of the colliding partons and the neutrino cannot be measured. The $\mathrm{W}$ mass is therefore extracted from 3 complementary observables transversal to the beam direction: $\mathrm{W}$ transverse mass $m_{T}$, electron $p_{T}^{e}$ and the missing $p_{T}^{v}$. The following relation can be derived: $\left.m_{T}=\sqrt{2 p_{T}^{e} p_{T}^{v}\left(1-\cos \Delta \varphi_{e v}\right.}\right)$. Those variables have different sensitivity to the same effects. They are not completely correlated and can be used in the statistical combination. For example the electron $p_{T}^{e}$ is most affected by the transverse motion of $\mathrm{W}\left(p_{T}^{W}\right)$, whereas transverse mass of $\mathrm{W}\left(m_{T}\right)$ is less sensitive to this effect, but more sensitive to the detector resolution effects (red dots) as can be seen from Fig. 3.
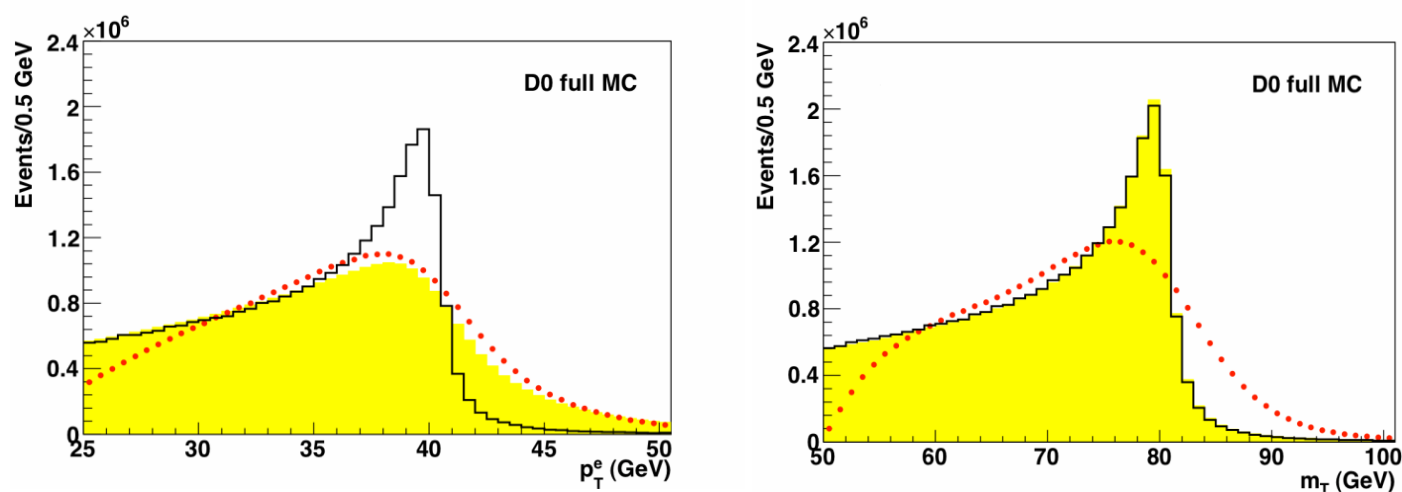

Fig.3 Different sensitivity of different variables to the same effects. Black histogram shows the distributions without any $p_{T}^{W}$, yellow histograms shows when $p_{T}^{W}$ is included. Red dots correspond to the inclusion of detector resolution effects.

Events are selected using a trigger requiring at least one electromagnetic (EM) cluster found at $|\eta|<1.05$, i.e. in the central calorimeter (CC), and with the energy threshold varying between 25 and $27 \mathrm{GeV}$. In the offline selection of $W \rightarrow e v$ event candidates we require matching in $(\eta, \varphi)$ space of at least one EM cluster in CC to a good track including at least one SMT hit and $p_{T}>10 \mathrm{GeV}$. Electron candidate with $p_{T}^{e}>25 \mathrm{GeV}$ has to pass shower shape and isolation criteria and at the same time has to point to the central $80 \%$ in azimuth of a CC module. Additional event criteria are $\mathbb{E}_{T}>25 \mathrm{GeV}, u_{T}<15 \mathrm{GeV}$, and $50<m_{T}<200 \mathrm{GeV}$. Transversal hadronic energy $u_{T}$ is the magnitude of the vector sum of the transverse components of the energies measured in calorimeter cells excluding those associated with the reconstructed electron. Missing momentum due to the escaping neutrino is defined as $\overrightarrow{\mathrm{E}}_{\mathrm{T}}=$ $-\left(\vec{p}_{T}^{e}+\vec{u}_{T}\right)$.

\subsection{Electromagnetic Energy Model and its Calibration}

As the properties of $\mathrm{Z}$ boson are known with very high precision from LEP measurements, $Z \rightarrow e e$ events are used to calibrate the EM calorimeter response. Detailed model with corrections for dead material, underlying events and noise is built. Corresponding calibration constants are determined from fits to $Z \rightarrow e e$ mass spectrum and to the electrons angular distributions. Example of consistency check for data divided into different luminosity 
bins shows good agreement between calibration constants (Fig. 4 - central values and one standard deviation contours of the fits to electron energy scale and offset). Energy scale is measured over broad energy range using boosted Z's. Final closure test is done with full MC. Electron energy resolution is driven by two components, the sampling fluctuations and the constant term. We get very good agreement between data and Parametrized MC Simulation (PMCS) for both terms (Fig.5).

\subsection{Hadronic Recoil Model and its Calibration}

For the recoil calibration a MC model with $Z \rightarrow v v$ events is used. Real $Z \rightarrow e e$, Minimum Bias (MB) and Zero Bias (ZB) data are used for the tuning of the momentum imbalance $\eta_{\mathrm{Imb}}$ with standard UA2 parameters [4]. The $\eta_{\mathrm{Imb}}$ is a projection of vector $\vec{p}_{T}(e e)+\vec{u}_{T}$ on the axis bisecting the dielectron opening angle. Five tunable parameters allow adjustement of the response and the resolution. In this model we consider four different contributions to the $\vec{u}_{T}$ :

$$
\vec{u}_{T}=\vec{u}_{T}^{\text {Hard }}+\vec{u}_{T}^{\text {Soft }}+\vec{u}_{T}^{\text {Elec }}+\vec{u}_{T}^{\text {FSR }}
$$

Where $\vec{u}_{T}^{\text {Hard }}$ is the hadronic energy from the $\mathrm{W}$ recoil, $\vec{u}_{T}^{\text {Soft }}$ describes the soft hadronic activity from $\mathrm{ZB}$ and $\mathrm{MB}$ events, $\vec{u}_{T}^{\text {Elec }}$ is the recoil energy that was reconstructed under the electron cone, as well as energy from the electron leaked outside the cone and $\vec{u}_{T}^{F S R}$ is out-ofcone FSR that is reconstructed as hadronic recoil.

\subsection{Analysis Strategy}

Measurement is based on the comparison of $m_{T}, p_{T}^{e}$ and $p_{T}^{v}$ data distributions with templates generated at different $M_{W}$ in steps of $10 \mathrm{MeV}$. Templates samples are generated with ResBos [5] and Photos [6] generators. ResBos takes care for the production and decay kinematics of $\mathrm{W}, \mathrm{Z} / \boldsymbol{\gamma}^{*}$ samples and Photos for final state radiations up to 2 photons. $\mathrm{W}(\mathrm{Z}) \mathrm{GRAD}[6]$ generators are used for full QED corrections estimation. Detector efficiencies, energy response and energy resolutions are obtained from PMCS package. Generated particles passage through the detector simulated by PMCS uses parametric functions and binned look-up tables based on the detailed GEANT3 simulations. PMCS is fine-tuned using control data samples like $Z \rightarrow e e, \mathrm{ZB}$ and $\mathrm{MB}$ events.

We are using blinded analysis, i.e. $M_{W}$ from binned likelihood fits were deliberately offset by some unknown value. Results were unblinded only after completing all consistency checks for $\mathrm{W} / \mathrm{Z}$ events.

\subsection{Measurement Results}

To check the stability of our results the data were divided into statistically independent samples according to different criteria, e.g. the instantaneous luminosity, the data taking periods, or the electron $\eta$. The fit ranges for the $p_{T}^{e}, p_{T}^{v}, m_{T}^{W}$ or the exclusion region near CC module edges were varied. The results are stable to within the measurement uncertainty for each of these tests. Very good agreement of the PMCS results with the data measurements is 
indicated by the $\chi^{2}$ values calculated from the differences between the data and the simulation (Fig. 6).

The systematic uncertainties and prospects for their further reduction are shown in Tab 1. Main experimental sources due to the electron energy scale, the electron energy resolution and the hadronic recoil calibration are of the statistical nature, governed by the limited size of the $Z \rightarrow e e$ calibration sample. Theoretical uncertainties are dominated by PDFs and to a smaller degree by the EW radiative corrections.

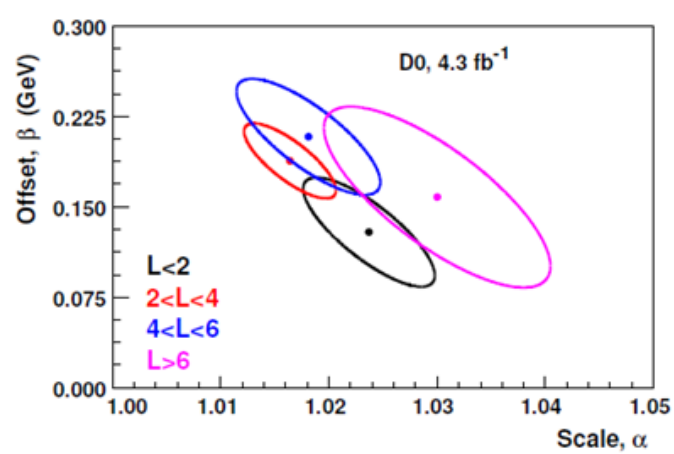

Fig. 4
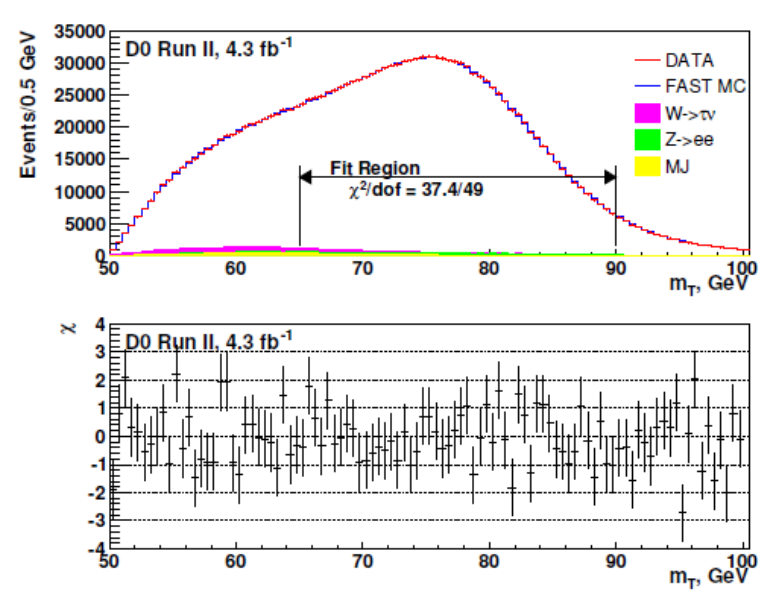

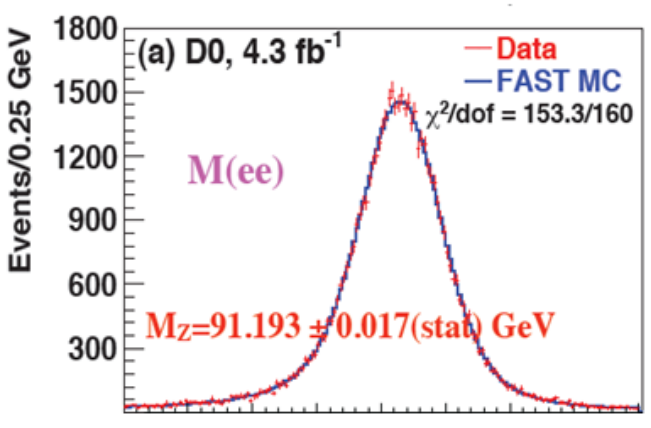

Fig. 5

\begin{tabular}{|c|c|}
\hline Method $\left(4.3 \mathrm{fb}^{-1}\right)$ & $M_{W}(\mathrm{MeV})$ \\
\hline$m_{T}(e, \nu)$ & $80371 \pm 13$ (stat) \\
\hline$p_{T}(e)$ & $80343 \pm 14$ (stat) \\
\hline $\mathbb{E}_{T}(e, \nu)$ & $80355 \pm 15$ (stat) \\
\hline Combination $m_{T} \oplus p_{T}\left(4.3 \mathrm{fb}^{-1}\right)$ & $80367 \pm 26($ syst + stat $)$ \\
\hline Combination $\left(5.3 \mathrm{fb}^{-1}\right)$ & $80375 \pm 23$ (syst + stat) \\
\hline
\end{tabular}

Fig. 6 


\subsection{Summary}

We have measured the $\mathrm{W}$ boson mass in $W \rightarrow e v$ channel with $4.3 \mathrm{fb}^{-1}$ of RunII DØ data with the precision of $\Delta M_{W}=26 \mathrm{MeV}$ (Tab. 1, column 2). Combination with $1 \mathrm{fb}^{-1}$ data from RunIIa results in the precision from single experiment which is equal to the previous world average of $\Delta M_{W}=23 \mathrm{MeV}$. The prospects for further improvements with the full RunII data set of about $10 \mathrm{fb}^{-1}$ are $\Delta M_{W}=19 \mathrm{MeV}$, when using only central electrons (Tab.1, column 4). Extending the measurement up to $\eta \rightarrow 2.5$, i.e. including the electrons in the calorimeter end cap (Tab.1, column 5), would bring down the precision to $\Delta M_{W}=15 \mathrm{MeV}$, which is the current world average. Further improvement of the $\Delta M_{W}$ measurement will be an important contribution to our better understanding of $\mathrm{EW}$ interactions.

\begin{tabular}{|c|c|c|c|c|c|}
\hline Source & $\begin{array}{l}\text { Public. } 2009 \\
\left(1.0 \mathrm{fb}^{-1}\right)\end{array}$ & $\begin{array}{l}\text { Public. } 2012 \\
\left(4.3 \mathrm{fb}^{-1}\right)\end{array}$ & $\begin{array}{l}\text { Proj. } \\
10 \mathrm{fb}^{-1}\end{array}$ & $\begin{array}{c}\text { Proj. } \\
10 \mathrm{fb}^{-1} \text { improv. }\end{array}$ & $\begin{array}{l}\text { Proj. } 10 \mathrm{fb}^{-1} \\
\text { improv. + EC }\end{array}$ \\
\hline Statistical & 23 & 13 & 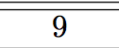 & 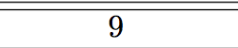 & 8 \\
\hline \multicolumn{6}{|l|}{ Experimental syst. } \\
\hline Electron energy scale & 34 & 16 & 11 & 11 & 10 \\
\hline Electron energy resolution & 2 & 2 & 2 & 2 & 2 \\
\hline EM shower model & 4 & 4 & 4 & 2 & 2 \\
\hline Electron energy loss & 4 & 4 & 4 & 2 & 2 \\
\hline Hadronic recoil & 6 & 5 & 3 & 3 & 2 \\
\hline Electron ID efficiency & 5 & 1 & 1 & 1 & 1 \\
\hline Backgrounds & 2 & 2 & 2 & 2 & 2 \\
\hline Subtotal experimental syst. & 35 & 18 & 13 & 12 & 11 \\
\hline \multicolumn{6}{|l|}{$\begin{array}{l}W \text { production } \\
\text { and decay model }\end{array}$} \\
\hline $\mathrm{PDF}$ & 9 & 11 & 11 & 11 & 5 \\
\hline QED & 7 & 7 & 7 & 3 & 3 \\
\hline boson $p_{T}$ & 2 & 2 & 2 & 2 & 2 \\
\hline Subtotal $W$ model & 12 & 13 & 13 & 12 & 6 \\
\hline Total systematic uncert. & 37 & 22 & 19 & 17 & 13 \\
\hline Total & $\overline{444}$ & $\begin{array}{l}\mathbf{2 6} \\
\text { Tab.1 }\end{array}$ & 21 & 19 & $\overline{15}$ \\
\hline
\end{tabular}

\section{References}

[1] V.M. Abazov et al. (DØ Collaboration), Phys.Rev.Lett. 103, 141801 (2009)

[2] V.M. Abazov et al. (DØ Collaboration), Phys.Rev.Lett. 108, 151804 (2012)

[3] arXiv:1305.3929v1 [hep-ex] 16 May 2013

[4] J.Alitti et al. (UA2 Collaboration), Phys.Lett. B 276, 354(1992)

[5] C.Balazs and C.P.Yuan, Phys.Rev. D 56, 5558 (1997)

[6] P.Golonka and Z.Was, Eur.Phys.J. C 45, 97(2006)

[7] U.Baur, S.Keller and D.Wackeroth, Phys.Rev. D 59013002 (1998) 